\title{
Candida albicans
}

National Cancer Institute

\section{Source}

National Cancer Institute. Candida albicans. NCI Thesaurus. Code C77170.

A species of diploid fungus in the phylum Ascomycota which is capable of mating but not of meiosis, and is a causal agent of opportunistic oral and genital infections in humans. 